\title{
Isolated unilateral trismus as a presentation of Chiari malformation: case report
}

\author{
Michelle Feinberg, MD, ${ }^{1}$ Parker Babington, MD, ${ }^{1}$ Shawn Sood, MD, MBA, ${ }^{1}$ and Robert Keating, MD ${ }^{2}$ \\ 'Department of Neurosurgery, The George Washington University School of Medicine; and 'Department of Neurosurgery, \\ Children's National Medical Center, Washington, DC
}

\begin{abstract}
The authors present a case of Chiari malformation manifesting as isolated trismus, describe the typical symptoms associated with Chiari malformation, and discuss the potential anatomical causes for this unique presentation. A 3-year-old boy presented with inability to open his jaw for 6 weeks with associated significant weight loss. The results of medical and radiological evaluation were negative except for Type I Chiari malformation with cerebellar tonsils $12 \mathrm{~mm}$ below the level of the foramen magnum. The patient underwent Chiari decompression surgery. Postoperatively, his ability to open his mouth was significantly improved, allowing resumption of a regular diet. Postoperative MRI revealed almost complete resolution of the syringobulbia. To the best of the authors' knowledge, this is the first reported case of isolated trismus from Chiari malformation with syringobulbia.
\end{abstract}

http://thejns.org/doi/abs/10.3171/2015.7.PEDS1592

KEY WORDS trismus; Chiari malformation; syringobulbia

C HIARI malformation Type I consists of caudal displacement of the cerebellar tonsils into the upper cervical spinal canal. Syringomyelia is commonly associated with the malformation. Patients with Chiari malformation can present with a multitude of symptoms, including but not limited to headache or neck pain, weakness or numbness in the extremities, ophthalmological or otological disturbances, and lower cranial nerve findings. ${ }^{15}$ Trismus, also known as masseter muscle spasm or lockjaw, is restricted mouth opening due to muscular dysfunction. It most commonly occurs in pediatric patients as the presenting symptom in Clostridium tetani infection. ${ }^{1}$ We present what is to our knowledge the first reported case of isolated trismus as the presenting symptom of Chiari I malformation.

\section{Case Report}

History

A 3-year-old healthy male child with no prior medical history suddenly developed the inability to open his jaw 6 weeks prior to presentation. At rest, his jaw was clearly deviated to the right, and with effort, he could minimally open his jaw on the left side. The jaw could not be actively opened by the examiner. No inciting event, including trauma or infection, could explain his current symptoms. Due to the trismus and the resulting inability to eat solid food, the patient had an 11-pound weight loss. He denied any jaw pain or headache. Results of his neurological examination, including evaluation of cranial nerve function, cerebellar signs, reflexes, and gait, were completely normal aside from the limitation of his jaw. He had normal facial sensation, including the $\mathrm{V} 3$ branch of the trigeminal nerve. There was no history of any hyperacusis or swallowing difficulty once he was able to get food into his mouth.

The initial workup focused on mechanical issues that could potentially limit motion of the jaw; however, no mechanical cause of trismus was identified. MRI of the brain and spine (Fig. 1) demonstrated a Type I Chiari malformation with the cerebellar tonsils $12 \mathrm{~mm}$ below the level of the foramen magnum. There was also an extensive holocord syrinx from the level of T-8 extending superiorly into the right dorsolateral medulla and right pontine tegmentum at the level of the pontomesencephalic junction. It was felt that the syringobulbia in the tegmentum could be contributing to a hyperactivity of the trigeminal nucleus, which could, in turn, be promoting unilateral trismus. 

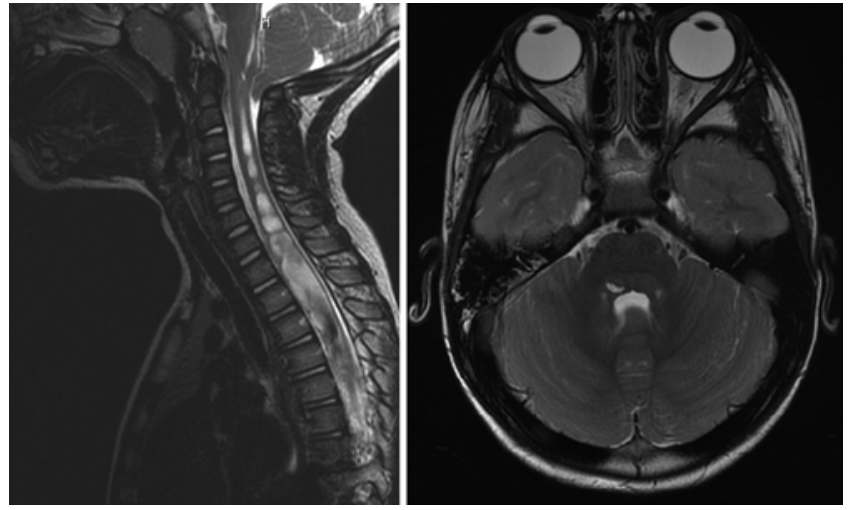

FIG. 1. Preoperative sagittal (left) and axial (right) T2-weighted MR images showing Chiari Type I malformation with holocord syrinx and syringobulbia.

\section{Operation}

In the absence of any other plausible etiological explanation, the patient was brought to the operating room for a suboccipital craniectomy and C-1 laminectomy as part of a Chiari decompression. With the initial induction of anesthesia using benzodiazepines and propofol, there was a small decrease in muscle tone of the jaw, but the significant contraction remained and prevented complete opening of the mouth. It was not until full muscle paralysis was obtained that the jaw was able to be opened sufficiently to allow for endotracheal tube placement

The standard operating practice of the senior surgeon when performing Chiari decompressions is to proceed in a stepwise fashion-first completing a bony posterior fossa decompression and if that appears to be inadequate based on intraoperative ultrasonography, to then proceed with a duraplasty. At that time, the outlet of the fourth ventricle is explored for the presence of adhesions. If necessary, a fourth ventricle stent (PS Medical T tube) is placed to ensure patency of the CSF outflow. In this case, intraoperative ultrasonography clearly demonstrated beaked cerebellar tonsils with pulsations causing brainstem compression as well as perceived flow within the syringobulbia. Following the bony decompression, intraoperative ultrasound imaging demonstrated an equivocal reduction in the size of the syrinx. Nevertheless, a decision was made to open the dura and arachnoid mater to explore the fourth-ventricle outlet. At this time several arachnoid webs at the fourthventricle outflow were identified and partially lysed, with a subsequent fourth ventricle stent being placed to allow for normal unobstructed CSF flow. At the conclusion of the decompression procedure, ultrasound demonstrated a more rounded appearance of the cerebellar tonsils, and the syringobulbia was now difficult to appreciate.

\section{Postoperative Course}

The patient had an uncomplicated recovery from surgery, with near-immediate improvements in his symptoms. On postoperative Day 1, the range of motion in his jaw was significantly improved and he was able to open his jaw wide enough to eat solid foods for the first time in 6 weeks. By the time of his first follow-up appointment, 1 month after the operation, he was eating his normal diet and had regained all the weight he had previously lost. Follow-up MRI studies performed 3 and 9 months postoperatively (Fig. 2) continued to show a decrease in size of the syrinx with almost complete resolution of the syringobulbia. The patient continued to gain weight appropriately for his age. At 1-year follow-up, the patient continues to have significant improvement in his jaw, although it has not completely returned to its baseline. He does have mild spacticity in the masseter muscle with slight jaw deviation, which is still continuing to improve. Functionally, he has sufficient range of motion of his jaw to allow him to eat and speak normally. His face remains grossly symmetric and he is now able to completely stick out his tongue.

\section{Discussion}

Chiari malformations were first described by Hans Chiari in $1891,{ }^{15}$ and the first Chiari decompression procedure was performed by James Gardner in $1950.5^{5}$ Of the subtypes of Chiari malformations described, Type I is the most common; it is characterized by tonsillar herniation greater than $5 \mathrm{~mm}$ inferior to McRae's line with no associated brainstem herniation or supratentorial anomalies. ${ }^{10}$ While headache and syringomyelia are commonly associated with Chiari I malformations, lower cranial nerve dysfunction is less common and occurs in only $20 \%$ of patients. ${ }^{1}$ This most commonly manifests as vocal cord paralysis, dysarthria, hoarseness, palatal weakness, sleep apnea, tongue atrophy, recurrent aspiration, and downbeating nystagmus. ${ }^{11,15}$

At our institution, Children's National Medical Center, over 140 Chiari decompressions have been performed over the past 10 -year period. During that same time pe-
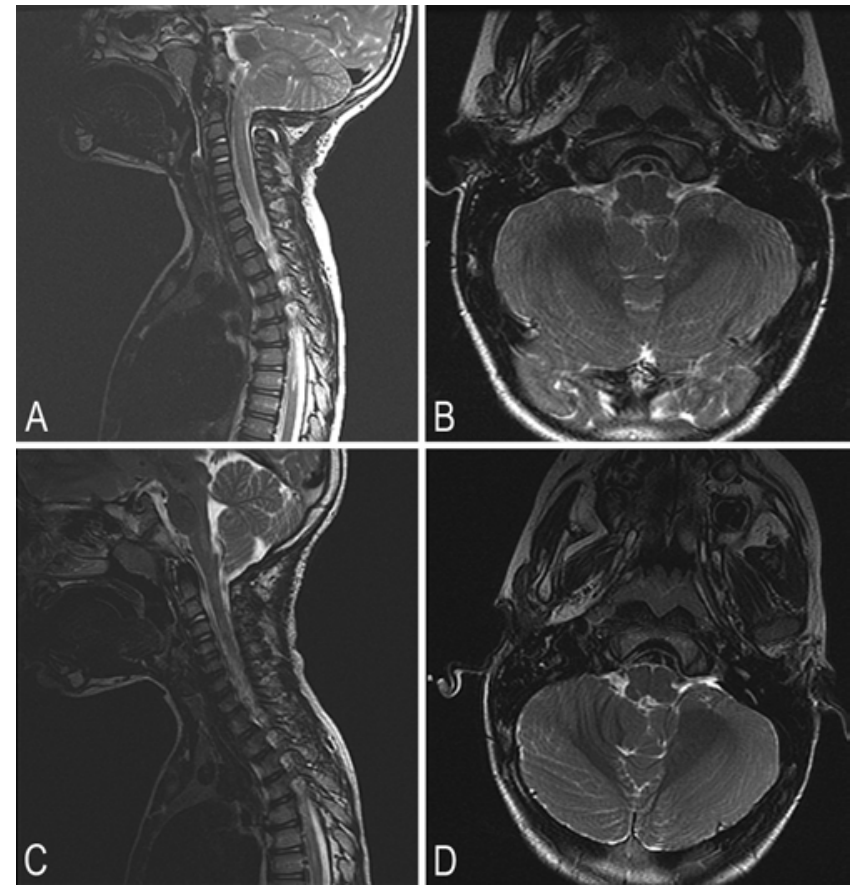

FIG. 2. Three-month postoperative sagittal (A) and axial (B) and 9-month postoperative sagittal (C) and axial (D) T2-weighted MR images showing significant improvement in both the syrinx and syringobulbia. 
riod, another 570 patients have been followed for incidentally found Chiari malformations and have remained asymptomatic, not requiring surgical intervention. As expected, the majority of our patients presented with headaches or neck pain, but there were a significant number of children with lower cranial nerve abnormalities. In our series of surgically treated cases, there were 13 children with central sleep apnea and another 12 with snoring-in all 25 cases, these abnormalities were caused by Chiari malformations and improved following decompression. Dysarthria, hoarseness, stridor, drooling, and recurrent aspiration were the other cranial nerve abnormalities seen.

Unlike the lower cranial nerves that exit from the medulla, the trigeminal nerve exits at the level of the pons. Due to its more cranial location, the trigeminal nerve is not typically affected by Chiari malformations. While there have been a few reports of trigeminal neuralgia associated with Chiari malformations, ${ }^{8,9}$ there have been no reports of any isolated trigeminal motor nerve dysfunction.

The trigeminal nerve is a mixed nerve that originates from the midlateral surface of the pons as a large sensory root and a smaller motor root. The motor component of the trigeminal nerve receives its input from corticobulbar fibers from the precentral gyrus through the corona radiata, internal capsule, and cerebral peduncle. ${ }^{2}$ It then decussates at the level of the pons to supply the trigeminal motor nuclei located on the floor of the fourth ventricle. ${ }^{2}$ The motor axons initially exit the pons anterosuperomedially to the sensory root, coursing anteriorly in between the middle cerebellar penduncle and the petrous bone through the cerebellopontine angle cistern. ${ }^{6}$ The nerve then crosses over the petrous apex at the trigeminal impression to travel inferiorly, under the petroclinoid ligament, to enter Meckel's cave. ${ }^{6}$ There, the motor root joins the sensory fibers of the mandibular division of the trigeminal nerve and exits through the foramen ovale.

The motor component continues peripherally with the sensory mandibular fibers to supply the muscles of mastication, including the masseter, temporalis, medial pterygoid, and lateral pterygoid as well as the tensor tympani and tensor veli palatine ${ }^{3,7}$ (Fig. 3).

Most commonly, trigeminal nerve disturbances associated with Chiari malformations manifest as trigeminal neuralgia, with at least 20 reported cases in the literature. ${ }^{8,13}$ Trigeminal neuralgia caused by Chiari malformation was first described by Dandy in $1934 .{ }^{4}$ Although the exact mechanism is unclear, 4 hypotheses have been proposed. ${ }^{8,13}$ The first theory is that brainstem displacement causes extraaxial elongation of the trigeminal nerve root, causing demyelination and remyelination in trigeminal sensory fibers that result in the formation of artificial synapses..$^{14}$ The second is that cerebellar tonsillar herniation can cause compression of the spinal trigeminal pathway, which contains pain-sensitive fibers. ${ }^{9}$ Another theory is that a cervical syrinx may cause indirect vascular compression or stretching or the trigeminal nerve at the nerve root entry zone. ${ }^{12}$ Finally, the Chiari malformation may cause micro-ischemic changes, which have been shown to cause trigeminal neuralgia in association other lesions. ${ }^{8}$ Approximately two-thirds of the patients described in the literature had sustained pain relief following Chiari de-

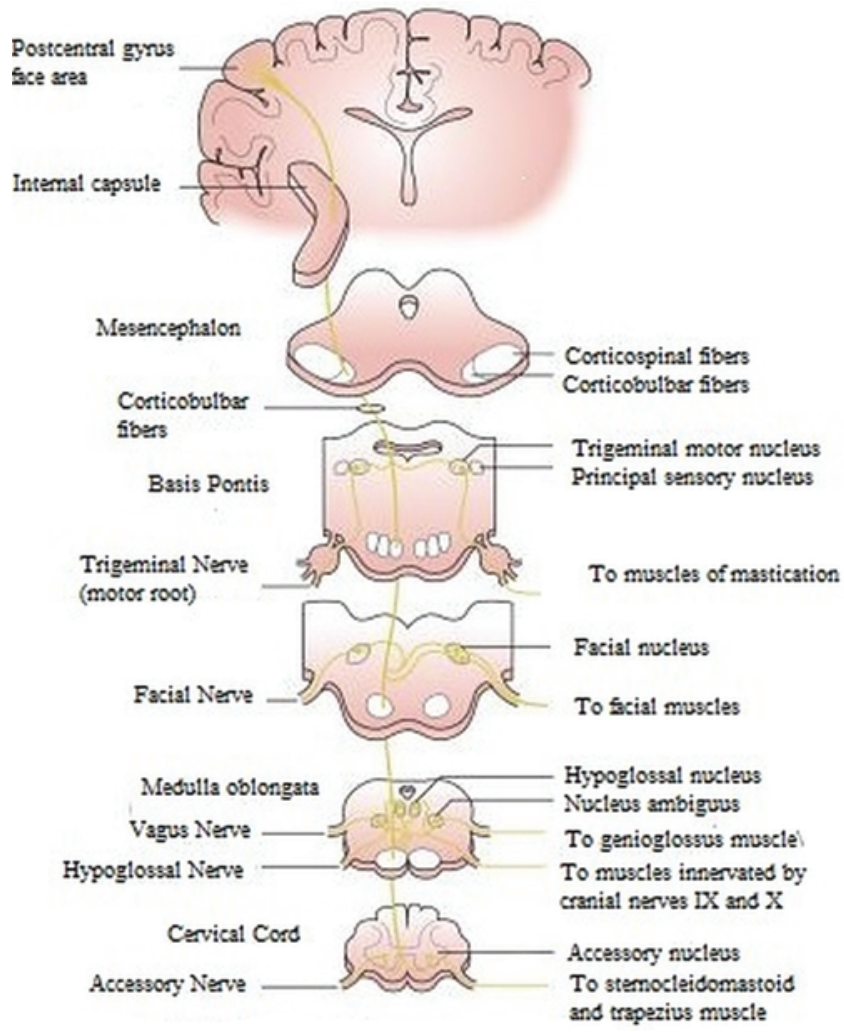

FIG. 3. A simplified version of the trigeminal nerve anatomy. Reproduced from Brazis P, Masdeu J, Biller J: Localization in Clinical Neurology, 6th ed. Philadelphia: Lippincott Williams \& Wilkins, 2011, p 7. Published with permission. Figure is available in color online only.

compression. ${ }^{8}$ Whether these theories are applicable in our patient's case is open for speculation. It is also possible that the expanding syringobulbia irritated the trigeminal motor nucleus, in turn causing a constant focus of irritation leading to trismus. It is interesting to note that the patient manifested an immediate reduction in trismus following surgery and a real-time decrease in the size of the syrinx, as demonstrated by intraoperative ultrasonography.

\section{Conclusions}

Chiari malformations are a common neurosurgical condition in both the pediatric and adult populations. Although the symptomatology has been well described in the literature, trigeminal nerve involvement is exquisitely rare and most commonly takes the form of trigeminal neuralgia. We present the first known case of a patient presenting with trismus as the only symptom of a Chairi malformation. We hypothesize that the syringobulbia caused by the Chiari malformation resulted in irritation of the trigeminal motor nucleus and subsequent masseter muscle spasticity. Although only an association and not true causation can be concluded, our patient did have a successful resolution of his symptoms as well as radiological improvement of the syrinx following a posterior fossa decompression.

\section{References}

1. Arnon S: Tetanus, in Kliegman RM, Stanton B, St. Gerne J, 
et al (eds): Nelson Textbook of Pediatrics. New York: Saunders, 2011, pp 991-994

2. Bathla G, Hegde AN: The trigeminal nerve: an illustrated review of its imaging anatomy and pathology. Clin Radiol 68:203-213, 2013

3. Brazis PW, Masdeu JC, Biller J: Cranial nerve V (the trigeminal nerve), in: Localization in Clinical Neurology. Philadelphia: Lippincott Williams \& Wilkins, 2011, pp 305-320

4. Dandy WE: Concerning the cause of trigeminal neuralgia. Am J Surg 24:447-455, 1934

5. Gardner WJ, Goodall RJ: The surgical treatment of ArnoldChiari malformation in adults; an explanation of its mechanism and importance of encephalography in diagnosis. J Neurosurg 7:199-206, 1950

6. Morton DA, Foreman KB, Albertine KH: Cranial nerves, in: The Big Picture: Gross Anatomy. New York: McGrawHill, 2011, pp 195-218

7. Naidich T, Duvernoy H, Delman B, Sorenson G, Kollias S, Haacke EM: Surface anatomy of the brain stem and cerebellum, in: Duvernoy's Atlas of the Human Brain and Cerebellum. New York: Springer, 2009, pp 30-34

8. Papanastassiou AM, Schwartz RB, Friedlander RM: Chiari I malformation as a cause of trigeminal neuralgia: case report. Neurosurgery 63:E614-E615, 2008

9. Rosetti P, Ben Taib NO, Brotchi J, De Witte O: Arnold Chiari Type I malformation presenting as a trigeminal neuralgia: case report. Neurosurgery 44:1122-1124, 1999

10. Soleau S, Tubbs S, Oakes WJ: Chiari malformations, in Albright A, Pollack I Adelson P (eds): Principles and Practice of Pediatric Neurosurgery, ed 2. New York: Thieme Medical Publishers, 2008, pp 217-232

11. Steinbok P: Clinical features of Chiari I malformations. Childs Nerv Syst 20:329-331, 2004
12. Teo C, Nakaji P, Serisier D, Coughlan M: Resolution of trigeminal neuralgia following third ventriculostomy for hydrocephalus associated with Chiari I malformation: case report. Minim Invasive Neurosurg 48:302-305, 2005

13. Than KD, Sharifpour M, Wang AC, Thompson BG, Pandey AS: Chiari I malformation manifesting as bilateral trigeminal neuralgia: case report and review of the literature. J Neurol Neurosurg Psychiatry 82:1058-1059, 2011

14. Tortosa A, Arbizu T, Ferrán E, Peres Serra J: [Trigeminal neuralgia as the first manifestation in Arnold-Chiari deformity.] Neurologia 6:148-150, 1991 (Span)

15. Tubbs RS, Cohen-Gadol AA: Hans Chiari (1851-1916). J Neurol 257:1218-1220, 2010

\section{Disclosures}

The authors report no conflict of interest concerning the materials or methods used in this study or the findings specified in this paper.

\section{Author Contributions}

Conception and design: Feinberg, Keating. Acquisition of data: Feinberg. Analysis and interpretation of data: Feinberg, Keating. Drafting the article: all authors. Critically revising the article: Feinberg, Keating. Reviewed submitted version of manuscript: Feinberg, Babington, Keating. Approved the final version of the manuscript on behalf of all authors: Feinberg. Administrative/ technical/material support: Feinberg. Study supervision: Keating.

\section{Correspondence}

Michelle Feinberg, 2201 L St. NW, Washington, DC 20037. email: michlfeinberg@gmail.com. 